\title{
Z. Orthop. Unfall. 2011
}

\section{Z. Orthop. Unfall. 2011}

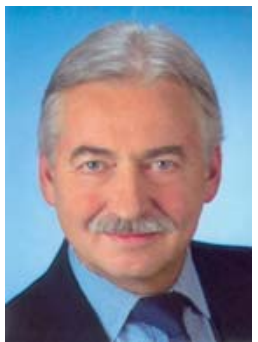

K. Weise

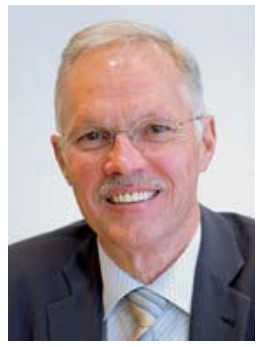

F. U. Niethard

\section{Bibliografie}

Dol http://dx.doi.org/

10.1055/s-0030-1270974

Z Orthop Unfall 2011; 149: 143-

144 ๑ Georg Thieme Verlag KG

Stuttgart . New York.

ISSN 1864-6697

Korrespondenzadressen Prof. Dr. med. Kuno Weise BG-Unfallklinik

Schnarrenbergstraße 95

72076 Tübingen

Tel.: 0 7071/606-1001

Fax: 07071/606-1002

weise@bgu-tuebingen.de

Prof. Dr. med. Fritz U. Niethard

Orthopädische Klinik

Universitätsklinik der

RWTH Aachen

Pauwelsstraße 30

52074 Aachen

Tel.: 0241/808-9410

Fax: 0241/808-2453

funiethard@googlemail.com

\section{Etwas gerne tun macht jede Last leichter.} Ovid

Dies ist das letzte vom Erstunterzeichnenden verantwortlich verfasste Editorial der Zeitschrift für Orthopädie und Unfallchirurgie. Das nunmehr feststehende, ab Mitte dieses Jahres aktiv in die Gestaltung der Zeitschrift einsteigende neue Herausgeberteam wird aus 2 Vertretern der uns nachfolgenden Generation von Orthopäden und Unfallchirurgen bestehen, die aufgrund ihrer unterschiedlichen fachlichen Schwerpunkte die vielfältigen Facetten unseres gemeinsamen Faches in ganzer Breite abdecken werden. Im letzten gemeinsamen Editorial für das nächste Heft der ZfOU werden die Herausgeber Näheres hierzu mitteilen und den Nachfolgern womöglich eine Art „Vermächtnis“ für die Zukunft der Zeitschrift mit auf den Weg geben. Wir sind fest davon überzeugt, dass der Verlag in Absprache mit uns die richtige Wahl für unsere Nachfolge in der Herausgeberschaft der Zeitschrift, die in den letzten Jahren einen festen Platz in der Landschaft orthopädisch-unfallchirurgischer Journale beansprucht hat, für deren erfolgreiche Zukunft getroffen hat. Anlässlich des Wechsels der verantwortlichen Herausgeber eines renommierten Publikationsorgans, welches sich vor ca. 4 Jahren aus 2 langjährig existierenden Fachjournalen mit orthopädischer (Zeitschrift für Orthopädie und ihre Grenzgebiete) bzw. unfallchirurgischer (Aktuelle Traumatologie) Ausrichtung entwickelt hat, muss eine bilanzierende Rückschau auf das bisher Geleistete erlaubt sein. Im allerersten gemeinsam verfassten Editorial der neu gegründeten ZfOU haben wir als Herausgeber ausgeführt, dass in Anlehnung an die Empfehlung der Fachgesellschaften, diese unter dem Motto „Einheit in der Vielfalt" unter Wahrnehmung von Synergien zusammenzuführen, auch die beiden im ThiemeVerlag erscheinenden Zeitschriften vereinigt werden könnten [1]. Mit der Gründung der ZfOU sollte die Bedeutung unserer Fachgebiete im Konzert deutschsprachiger wissenschaftlicher Journale gefestigt, womöglich herausgehoben und der vorgezeichnete Weg bis zur Gründung der DGOU im Juli 2008 publikatorisch begleitet werden. Den an einer Veröffentlichung in der ZfOU interessierten Autoren sollte die Möglichkeit offenstehen, dort ein geeignetes Forum von der wissenschaftlich anspruchsvollen Originalarbeit bis zur Darstel- lung außergewöhnlicher und spektakulärer Fälle vorzufinden. Hinsichtlich der von uns ins Auge gefassten Leserschaft sollte die Zeitschrift interessant, lehrreich und eine feste Größe für Informationen wissenschaftlicher und berufspolitischer Art bzw. solcher mit dem Hintergrund von Weiter- und Fortbildung sein.

Abwechselnd von der Herausgebern sowie von zu speziellen Fragestellungen aufgeforderten Autoren wurden Editorials verfasst, deren Fokus sich auf die genannten berufspolitischen Entwicklungen $[2,3]$, auf besondere Schwerpunktthemen [4-6], auf den weiteren Fortgang der Zusammenführung unserer Fachgesellschaften [7-9] und auf die spezielle Situation unseres Nachwuchses [1013] richtete. Zahlreiche Originalarbeiten zu aktuellen wissenschaftlichen Erkenntnissen, interessante Kasuistiken, grundlegende Arbeiten zur Fort- und Weiterbildung sowie die Rubrik „Orthopädie und Unfallchirurgie aktuell“ wurden in der ZfOU bis heute veröffentlicht und haben unsere Position im Reigen der Fachjournale mit entsprechender Ausrichtung gefestigt [14-16]. Eine solche Bewertung kann, bei aller Bescheidenheit, aus der ganz überwiegend positiven Resonanz und entsprechenden Äußerungen unserer geneigten Leserschaft während der vergangenen Jahre konstatiert werden.

Die Zeitschrift ist gut aufgestellt, das kann man zum Ende unserer Tätigkeit als Herausgeber festhalten. Ungeachtet der nicht geringen Konkurrenz einschlägiger Publikationsorgane mit orthopädisch-unfallchirurgischer Ausrichtung können wir auf eine eher zunehmende Anzahl von Manuskripteinreichungen zurückgreifen. An dieser Stelle möchten die Herausgeber allen bisherigen Autoren unseren ausdrücklichen Dank abstatten und der Zeitschrift auch für die Zukunft eine entsprechende Frequenz an eingereichten Arbeiten wünschen. Unser Dank gilt auch den Rubrikherausgebern für ihren geleisteten Beitrag, wobei sie die Zusammenarbeit mit den neuen Herausgebern ganz sicher in gleicher Weise fortführen werden. Dem Beirat der ZfOU, welcher schwerpunktmäßig für das Review-Verfahren verantwortlich zeichnet, sei für die in aller Regel gleichermaßen zeit- wie fachgerechte Beurteilung eingereichter Manuskripte gedankt; Fortsetzung folgt. Die Zusammenarbeit mit dem Thieme-Verlag, in Sonderheit mit Frau Maier, Frau Jürgens und Frau Stickel war ebenso erfolgreich wie ange- 
nehm und hat ganz wesentlich zum Gedeihen der Zeitschrift beigetragen. Ausdrücklich erwähnt werden soll nicht zuletzt auch die tatkräftige Unterstützung, die wir durch Herrn Sommer in Aachen sowie Herrn Dr. Stuby in Tübingen erfahren haben; ohne deren Hilfe wäre unsere Arbeit sehr viel schwieriger gewesen. $\mathrm{Zu}$ ganz besonderem Dank sind wir, last but not least, Ihnen liebe Leser verpflichtet, denn was wäre eine Fachzeitschrift ohne das von Ihnen entgegengebrachte Interesse an deren Inhalt.

\section{Es gibt keine Vergangenheit ohne Zukunft. \\ Egon Friedell}

\section{K. Weise}

F.U. Niethard

\section{Literatur}

1 Niethard FU, Weise K. Die neue Zeitschrift für Orthopädie und Unfallchirurgie - Einheit in der Vielfalt. Z Orthop Unfall 2007; 145: 111-112

2 Dreinhöfer KE. Die Bone and Joint Decade - Chancen für Orthopädie und Unfallchirurgie. Z Orthop Unfall 2007; 145: 399-402

3 Niethard FU, Weise K. Ökonomie in Orthopädie und Unfallchirurgie von der Qualitätssicherung zur Priorisierung. Z Orthop Unfall 2009; 147: 417

4 Albrecht D. Kreuzbandrekonstruktion. Z Orthop Unfall 2007; 145: 705
5 Niethard FU, Weise K. Iatrogene Schäden - Fehlervermeidung in Orthopädie und Unfallchirurgie. Z Orthop Unfall 2008; 146: 173

6 Weise K, Niethard FU. Das Aktionsbündnis Patientensicherheit: Beweggründe - Entstehung - derzeitiger Stand. Z Orthop Unfall 2010; 148: 639-640

7 Weise K, Hassenpflug J. Editorial. Z Orthop Unfall 2007; 145: 539

8 Niethard FU, Weise K. Ein historischer Tag - Deutsche Gesellschaft für Orthopädie und Unfallchirurgie gegründet. Z Orthop Unfall 2008; 146: 437-438

9 Niethard FU, Weise K. Orthopädisch-unfallchirurgische Forschung in Deutschland. Z Orthop Unfall 2010; 148: 143-144

10 Weise K, Niethard FU. Das junge Forum der DGOU - über die Wünsche und Vorschläge des Nachwuchses in Unfallchirurgie und Orthopädie. Z Orthop Unfall 2009; 147: 15-16

11 Weise K, Niethard FU. Zum Nachwuchsmangel in Unfallchirurgie und Orthopädie. Z Orthop Unfall 2010; 148: 17-18

12 Niethard FU, Weise K. Weiterbildung auf dem Prüfstand. Z Orthop Unfall 2010; 148: 385-386

13 Niethard FU, Weise K. Aus- und Weiterbildung im Fokus. Z Orthop Unfall 2011; 149: 15-16

14 Weise K, Niethard FU. Die Entwicklung der Zeitschrift für Orthopädie und Unfallchirurgie - aus Sicht der Herausgeber. Z Orthop Unfall 2008; 146: 311

15 Weise K, Niethard FU. Editorial. Z Orthop Unfall 2008; 146: 579

16 Niethard FU, Weise K. Die ganze Breite des Faches. Z Orthop Unfall 2008; 146: 709 